\title{
Exosomes from triple-negative breast cancer cells can transfer phenotypic traits representing their cells of origin to secondary cells
}

\author{
Keith O'Brien ${ }^{\mathrm{a}}$, Sweta Rani ${ }^{\mathrm{a}}$, Claire Corcoran ${ }^{\mathrm{a}}$, Robert Wallace ${ }^{\mathrm{a}}$, Linda Hughes ${ }^{\mathrm{b}}$, \\ Anne M. Friel ${ }^{a}$, Susan McDonnell ${ }^{\mathrm{b}}$, John Crown ${ }^{\mathrm{c}}$, Marek W. Radomski ${ }^{\mathrm{a}}$, \\ Lorraine O’Driscoll ${ }^{\mathrm{a}, *}$
}

${ }^{a}$ School of Pharmacy and Pharmaceutical Sciences \& Trinity Biomedical Sciences Institute, Trinity College Dublin, Dublin 2, Ireland

${ }^{\mathrm{b}}$ School of Chemical and Bioprocess Engineering, University College Dublin, Dublin 4, Ireland

${ }^{c}$ Molecular Therapeutics for Cancer Ireland, Dublin City University, Dublin 9, Ireland

\section{KEYWORDS \\ Exosomes \\ Breast cancer \\ Triple-negative breast cancer \\ Cell-to-cell communica- \\ tion \\ Epigenetic}

\begin{abstract}
Background: Triple-negative breast cancer (TNBC) accounts for $15-20 \%$ of breast cancers but is responsible for a disproportionate number of deaths. We investigated the relevance, in TNBC, of nano-sized exosomes expelled from cells. Specifically, we compared effects of exosomes derived from the claudin-low TNBC cell line Hs578T and its more invasive Hs578Ts(i) $)_{8}$ variant, as well as exosomes from TNBC patient sera compared to normal sera. Methods: Exosomes were isolated from conditioned media (CM) of Hs578T and Hs578Ts(i) cells and from sera by filtration and ultracentrifugation. Successful isolation was confirmed by transmission electron microscopy and immunoblotting. Subsequent analysis, of secondary/ recipient cells in response to exosomes, included proliferation; motility/migration; invasion; anoikis assays and endothelial tubule formation assays.

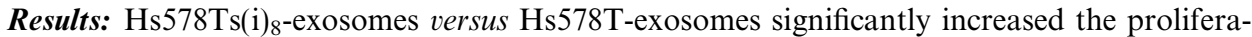
tion, migration and invasion capacity of all three recipient cell lines evaluated i.e. SKBR3, MDA-MB-231 and HCC1954. Exosomes from Hs578Ts(i) $)_{8}$ cells also conferred increased invasiveness to parent Hs578T cells. Hs578Ts(i) $)_{8}$-exosomes increased sensitivity of SKBR3, MDA-MB-231 and HCC1954 to anoikis when compared to the effects of Hs578T-exosomes reflecting the fact that $\mathrm{Hs}_{578 \mathrm{Ts}}(\mathrm{i})_{8}$ cells are themselves innately more sensitive to anoikis. In

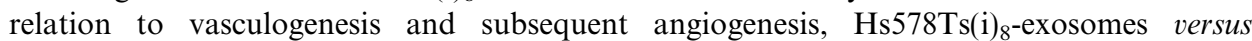
Hs578T-exosomes stimulated significantly more endothelial tubules formation. Finally, our
\end{abstract}

\footnotetext{
* Corresponding author: +35318962822 .

E-mail address: lodrisc@tcd.ie (L. O’Driscoll).
} 
pilot translational study showed that exosomes from TNBC patients' sera significantly increased recipient cells' invasion when compared to those derived from age- and gendermatched healthy control sera.

Conclusion: This study supports the hypothesis that TNBC exosomes may be involved in cancer cell-to-cell communication, conferring phenotypic traits to secondary cells that reflect those of their cells of origin.

(C) 2013 Elsevier Ltd. All rights reserved.

\section{Introduction}

Exosomes and microvesicles expelled from eukaryotic cells have attracted much recent interest since the realisation that these are not merely debris, but rather miniature maps of their cells of origin with both physiological and pathological relevance. ${ }^{1}$ Accumulating evidence indicates that exosomes $(\sim 30-120 \mathrm{~nm})$ and microvesicles (up to $1000 \mathrm{~nm}$ ) may play an important role in cell-to-cell communication. $^{2-4}$ In cancer, these entities have been implicated in carcinogenesis and tumour growth; moulding the tumour microenvironment; promoting angiogenesis and modulating immune response. ${ }^{5-9}$

Triple-negative breast cancers (TNBCs), although constituting a minority of breast cancers (15-20\%), cause a disproportionate number of metastatic cases and breast cancer deaths. TNBCs, which have $\sim 70-80 \%$ overlap with the basal subtypes, ${ }^{10,11}$ are classified by their lack of expression of oestrogen receptor (ER) and progesterone receptor (PR) and neither over-expression nor amplification of human epidermal growth factor receptor 2 (HER2) gene. This substantially limits treatment options because of their insensitivity to widely used targeted approaches such as endocrine therapies and trastuzumab that have proven effective at reducing breast cancer mortality. Additionally, compared to other breast tumours, TNBCs occur most frequently in younger patients ( $<50 \mathrm{yrs}$ ), and those tumours tend to be of higher-grade at presentation with more aggressive behaviour. These cancers typically relapse with distant metastases rather than with local, more manageable, recurrence and have increased likelihood of developing visceral metastases including central nervous involvement. TNBC is associated with shortened disease-free interval post-surgery and post-adjuvant treatment and, ultimately, a shortened overall survival. ${ }^{12-15}$ Furthermore, TNBCs are themselves a heterogeneous group within which the claudinlow subtype is associated with worst prognosis. ${ }^{16}$

Compared to the overall prevalence of breast cancer, a limited number of reports have indicated exosomes and/or microvesicles to be of relevance in breast cancer. $^{17-19}$ The focus, however, has typically been on HER2-overexpressing cells and their exosomes. Here, in what we believe to be the first study specifically considering exosomes in the context of TNBC, we have included analysis of exosomes from the claudin-low TNBC cell line Hs $578 \mathrm{~T}^{20}$ and its isogenic subclone,
Hs578Ts(i) ${ }_{8},{ }^{21}$ as well as exosomes from TNBC patients compared to healthy controls. Hs $578 \mathrm{Ts}(\mathrm{i})_{8}$, compared to Hs578T parent cells, has the same genetic background but significantly increased capacity to proliferate, migrate, invade through extracellular matrix and produce tumours in mice. This cell line pair is, therefore, very useful for investigating the capabilities of exosomes to transfer phenotypic traits representative of their cells of origin to secondary recipient cells.

\section{Materials and methods}

\subsection{Cell culture}

All cell lines are from breast tissue and were obtained from ATCC and cultured at $37^{\circ} \mathrm{C} / 5 \% \mathrm{CO}_{2}$. Hs578T and it's isogenic subclone, Hs578Ts(i) $)_{8}$ were grown in Dulbecco's modified Eagle's medium (DMEM) (Sigma-Aldrich), $10 \%$ foetal bovine serum (FBS) (PAA), $2 \mathrm{mM}$ L-glutamine (Sigma-Aldrich), and $10 \mu \mathrm{g} /$ $\mathrm{ml}$ of insulin (Sigma-Aldrich). SKBR3, MDA-MB231, and HCC1954 were grown in RPMI-1640 (Sigma-Aldrich), 10\% FBS, 2 mM L-glutamine.

\subsection{Exosome isolation}

\subsubsection{Conditioned medium (CM)}

Hs578T and Hs578Ts $(\mathrm{i})_{8}$ were seeded $\left(1 \times 10^{5}\right.$ cells/ flask) in 9xT75 flasks in complete medium overnight. Medium was then replaced with DMEM containing $10 \%$ exosome-depleted-FBS (dFBS; depletion of exosomes as we previously described ${ }^{22}$ ), $2 \mathrm{mM}$ L-Glutamine, $1 \%$ penicillin/streptomycin (Sigma-Aldrich), $10 \mu \mathrm{g} / \mathrm{ml}$ insulin. Cells were cultured for 5 days to $\sim 80-90 \%$ confluency. Exosomes were subsequently isolated from $\mathrm{CM}$ as previously detailed. ${ }^{22}$ In brief, CM was centrifuged at $2000 \mathrm{~g}$ for $10 \mathrm{~min}$ at $4{ }^{\circ} \mathrm{C}$ prior to filtering to eliminate cells and dead cells; then filtered using $0.45 \mu \mathrm{m}$ pore filters and subsequently ultracentrifuged at $110,000 \mathrm{~g}$ for $75 \mathrm{~min}$ at $4{ }^{\circ} \mathrm{C}$. Exosome pellets were washed in phosphate buffered solution (PBS) and ultracentrifugation repeated. Exosome quantities used are detailed in Section 3.

\subsubsection{Human serum specimens}

Serum specimens were obtained from Asterand biobank. As for CM, exosomes were isolated from $250 \mu 1$ 
aliquots of serum from TNBC patients and agedmatched healthy controls $(n=12)$. Information on 10 TNBC and 10 control donors is summarised in Table 1. Information currently accessible on the other two pairs of TNBC and control sera is age only; which was approximately $75 \mathrm{yrs}$ and approximately $55 \mathrm{yrs}$, respectively.

\subsection{Immunoblotting}

Total cellular proteins were extracted from cells and exosomes using sodium dodecyl sulphate (SDS) lysis buffer (250 nM Tris-HCl, pH 7.4; 2.5\% SDS). Proteins (20-30 $\mu \mathrm{g})$ were resolved on $12.5 \%$ SDS gels and transferred onto polyvinylidene difluoride membranes (Bio$\mathrm{Rad})$. Blots were blocked in $5 \%(\mathrm{w} / \mathrm{v})$ non-fat milk in $\mathrm{PBS} / 0.1 \%$ Tween and incubated overnight at $4{ }^{\circ} \mathrm{C}$ with primary antibodies to PDC6I/Alix (1:1000; Abcam), TSG101 (1:1000; Abcam), CD63 (1:500; Invitrogen); Claudin 1 (1:200; Invitrogen); Claudin 3 (1:200; Invitrogen); Claudin 4 (1:400; Invitrogen); and Claudin 5 (1:200; Invitrogen). Secondary antibodies were incubated for $1 \mathrm{~h}$ at room temperature. Blots were developed as previously described. ${ }^{22}$

\subsection{Transmission electron microscopy}

A $\sim 10 \mu 1$ aliquot of exosomes was placed onto parafilm (Sigma-Aldrich). A 300 mesh copper grid (Agar Scientific) was placed on top of the drop for $45 \mathrm{~min}$. The grid was subsequently washed thrice in $0.05 \mathrm{M}$ phosphate buffer (freshly prepared using dihydrogen potassium phosphate (Sigma-Aldrich) and dipotassium hydrogen phosphate (Merck)) for 5 min, fixed in 3\% glutaraldehyde (Agar Scientific) for $10 \mathrm{~min}$, washed thrice for $5 \mathrm{~min}$ in $\mathrm{dH}_{2} \mathrm{O}$ and contrasted in $2 \%$ uranyl acetate $(\mathrm{BDH})$. Grids were examined at $100 \mathrm{kV}$ using a JEOL JEM-2100 TEM.

\subsection{Confocal microscopy}

Exosomes were labelled using PKH67GL (SigmaAldrich), following manufacturer's instructions, added to cells of interest and analysed by confocal microscopy. Briefly, $5 \times 10^{-6} \mathrm{M}$ of PKH67GL was added to $1 \mu \mathrm{g}$ exosomes for $3 \mathrm{~min}$. Staining was stopped with $2 \mathrm{ml}$ of FBS (1 min). Complete medium $(4 \mathrm{ml})$ was added and centrifuged at $100,000 \mathrm{~g}, 4^{\circ} \mathrm{C}, 30 \mathrm{~min}$; washed with complete medium; centrifuged at $100,000 \mathrm{~g}, 4^{\circ} \mathrm{C}$, for $1 \mathrm{~h}$, and the pellet was re-suspended in $100 \mu \mathrm{l}$ PBS. Secondary/recipient cells were labelled with $2 \mu \mathrm{M}$ CM-DiI (Molecular Probes, Invitrogen; probes.invitrogen.com) for $5 \mathrm{~min}$ at $37^{\circ} \mathrm{C}, 15 \mathrm{~min}$ at $4{ }^{\circ} \mathrm{C}$. Of note, manufacturer's instructions indicate that incubation at this lower temperature appears to allow the dye to label the cell membrane but slows down endocytosis, thus reducing the changes of dye localisation into cytoplasmic vesicles. These cells were then washed twice with PBS.

PKH67GL-labelled exosomes $(5 \mu \mathrm{g})$ were added to SKBR3 cells, incubated for $30 \mathrm{~min}$ at $4{ }^{\circ} \mathrm{C}$ and then for $1 \mathrm{~h}$ at $37^{\circ} \mathrm{C}$. The cells were subsequently fixed with $4 \%$ paraformaldehyde (Sigma-Aldrich), mounted with

Table 1

Information available on the individual triple-negative breast cancer (TNBC) patients and healthy control volunteers who gave consent to have serum specimens analysed.

\begin{tabular}{|c|c|c|c|c|c|c|c|c|c|}
\hline & Age (yr) & Tumour Stage & Height $(\mathrm{cm})$ & Weight (Kg) & BMI & $\begin{array}{l}\text { No. of } \\
\text { pregnancies }\end{array}$ & $\begin{array}{l}\text { No. of } \\
\text { live births }\end{array}$ & Smoking Status & Alcohol Status \\
\hline \multicolumn{10}{|c|}{ (A) TNBC patient } \\
\hline TNBC-1 & 55 & IIIB & 164 & 88 & 32.72 & 3 & 2 & Never used & Occasional use \\
\hline TNBC-2 & 61 & $\mathrm{I}$ & 167 & 78 & 27.97 & 2 & 2 & Never used & Occasional use \\
\hline TNBC-3 & 56 & IIA & 170 & 84 & 29.07 & 3 & 3 & Never used & Occasional use \\
\hline TNBC-4 & 58 & $\mathrm{I}$ & 170 & 68 & 23.53 & 0 & 0 & Never used & Never used \\
\hline TNBC-5 & 58 & IIA & 173 & 113 & 37.76 & 2 & 1 & Never used & Occasional use \\
\hline TNBC-6 & 61 & $\mathrm{I}$ & 167 & 78 & 27.97 & 2 & 2 & Never used & Occasional use \\
\hline TNBC-7 & 70 & IIB & 175 & 98 & 32.00 & 2 & 2 & Never used & Occasional use \\
\hline TNBC-8 & 72 & IIA & 172 & 81 & 27.38 & 6 & 3 & Never used & Occasional use \\
\hline TNBC-9 & 66 & IIA & 155 & 71 & 29.55 & 4 & 3 & Never used & Never used \\
\hline TNBC-10 & 67 & IIB & 170 & 88 & 30.45 & 4 & 3 & Never used & Occasional use \\
\hline \multicolumn{10}{|c|}{ (B) Healthy control } \\
\hline C-1 & 55 & NA & 163 & 65 & 24.46 & 7 & 5 & Never used & Occasional use \\
\hline $\mathrm{C}-2$ & 61 & NA & 160 & 68 & 26.56 & 3 & 3 & Never used & Current use \\
\hline $\mathrm{C}-3$ & 56 & NA & 170 & 74 & 25.61 & 4 & 3 & Never used & Never used \\
\hline $\mathrm{C}-4$ & 55 & NA & 166 & 73 & 26.49 & 4 & 2 & Current use & Occasional use \\
\hline C-5 & 56 & NA & 163 & 73 & 27.48 & 3 & 2 & Occasional use & Occasional use \\
\hline C-6 & 51 & NA & 160 & 68 & 26.56 & 3 & 3 & Never used & Current use \\
\hline C-7 & 70 & NA & 150 & 73 & 32.44 & 4 & 4 & Never used & Never used \\
\hline C-8 & 73 & NA & 170 & 120 & 41.52 & 1 & 1 & Never used & Never used \\
\hline C-9 & 56 & NA & 163 & 57 & 21.45 & 0 & 0 & Never used & Occasional use \\
\hline C-10 & 68 & NA & 157 & 66 & 26.78 & 1 & 1 & Never used & Never used \\
\hline
\end{tabular}


DAPI-containing ProLong Gold Anti-fade reagent (Invitrogen, United Kingdom (UK)), and assessed by Olympus FV1000 confocal microscope.

\subsection{Proliferation rate measurement}

Hs578T and Hs578Ts(i) $)_{8}$ cells $\left(5 \times 10^{3}\right.$ cells/well $)$ were seeded in 24-well plates. After $72 \mathrm{~h}$, cells were counted with a Neubauer haemocytometer. To assess if exosomes transfer proliferation characteristics representative of the differing Hs578T cell line variants, SKBR3, MDA-MB-231 and HCC1954 cells were seeded at $2 \times 10^{3}$ cells/well in 96-well plates, using $10 \% \mathrm{dFBS}$ medium and $2 \mu \mathrm{g} /$ well of either Hs578T-exosomes or Hs578Ts(i) $)_{8}$-exosomes. After $72 \mathrm{~h}$, cells were counted.

\subsection{Migration}

SKBR3, MDA-MB-231 and HCC1954 cells $\left(3 \times 10^{5}\right.$ cells/well $)$ were seeded in 24 -well plates and grown to confluency, scratched with a $200 \mu$ pipette tip, and $10 \mu \mathrm{g} /$ well of Hs578T-exosomes or Hs578Ts(i) 8 $^{-}$ exosomes were added. The wounded areas were monitored by phase contrast microscopy and measured using Olympus Cell^A Image Acquisition Software.

\subsection{Invasion assays}

Transwell inserts were coated with extracellular matrix (ECM) as previously described. ${ }^{23}$ Medium containing $10 \% \mathrm{dFBS}(500 \mu \mathrm{l})$ was added to each well. Hs578T $\left(5 \times 10^{4}\right)$, SKBR3 $\left(5 \times 10^{4}\right)$, MDA-MB-231 $\left(5 \times 10^{4}\right)$ or HCC1954 $\left(2.5 \times 10^{4}\right)$ cells, in $500 \mu \mathrm{l} \mathrm{com-}$ plete medium, were added to each insert. When cells had attached, medium within the inserts was replaced with medium containing $1 \% \mathrm{dFBS}$ and $15 \mu \mathrm{g}$ of Hs578T, Hs578Ts(i) 8 , or patients or control serumderived exosomes. After $72 \mathrm{~h}$, medium was removed, the inside of the insert was wiped with a PBS-Soaked Q-tip, and stained with $1 \%$ crystal violet (SigmaAldrich). The crystal violet was solubilised with 33\% glacial acetic acid and read at $570 \mathrm{~nm}$.

\subsection{Anoikis assays}

Hs578T and Hs578Ts(i) $)_{8}$ cells $\left(5 \times 10^{4}\right.$ cells/well $)$ were plated onto poly-hydroxyethyl methactylic acidcoated (p-HEMA; Sigma-Aldrich) 24-well plates - or $95 \%$ ethanol-coated control plates - and cultured for $24 \mathrm{~h}$. Alamar blue (100 $\mu \mathrm{l} /$ well) (Sigma-Aldrich) was added for $3.5 \mathrm{~h}$ and read at $570 \mathrm{~nm}$.

To assess if exosomes transfer anoikis characteristics to recipient cells representative of their cells of origin, SKBR3, MDA-MB-231 and HCC1954 cells $\left(5 \times 10^{4}\right.$. cells/well) were seeded onto p-HEMA-plates with
$10 \mu \mathrm{g}$ of Hs578T-exosomes or Hs578Ts(i) $)_{8}$-exosomes, cultured for $72 \mathrm{~h}$, and assessed as above.

\subsection{In vitro vasculogenesis and angiogenesis}

The effects of exosomes on vasculogenesis and angiogenesis were analysed using the V2a Kit (TCS-Cellworks) according to manufacturer's instructions. Briefly, human endothelial cells were cultured over a period of 14 days in a 24-well plate, where the cells were subjected to Hs578T-exosomes or Hs578Ts(i) -exo- $^{-}$ somes $(10 \mu \mathrm{g})$ treatment; added freshly every $48 \mathrm{~h}$. 14 days post seeding, cells were fixed with $70 \%$ ethanol. Tubules were stained for CD31 (PECAM-1) using an anti-CD31 antibody (1:400) (TCS-Cellworks). CD31 tubule visualisation was achieved with the addition of the insoluble substrate (BCIP/NBT), resulting in tubules forming a dark purple colour. Tubules were photographed, and quantitative measurement of tubule development (i.e. the combined total lengths of the network of tubules formed) was determined using ImageJ software 'skeletonise' function.

Tubules were photographed using ImageJ software to measure tubule formation

\subsection{Zymography}

Zymography to detect matrixmetalloprotease (MMP) activity was performed as previously described. ${ }^{24}$ In summary, total cellular proteins were extracted from cells and exosomes using SDS lysis buffer $(250 \mathrm{nM}$ Tris- $\mathrm{HCl}, \mathrm{pH} 7.4 ; 2.5 \%$ SDS $)$. Proteins $(10 \mu \mathrm{g})$ were electrophoresed on an SDS-PAGE containing $0.2 \%$ gelatin (Sigma). Gels were run on ice at $150 \mathrm{~V}$ for $2 \mathrm{~h}$ and were subsequently washed with $2.5 \%$ Triton, twice for $20 \mathrm{~min}$ each, rocking and at room temperature. Three 20 min washes were then performed using zymography buffer $\left(0.15 \mathrm{M} \mathrm{NaCl}, 5 \mathrm{mM} \mathrm{CaCl} 2,0.05 \% \mathrm{NaN}_{3}\right.$, and $50 \mathrm{mM}$ Tris- $\mathrm{HCl}$ buffer, $\mathrm{pH}$ 7.5), with fresh zymography buffer replaced after the final washing step. Gels were placed at $37^{\circ} \mathrm{C}$ in zymography buffer for $48 \mathrm{~h}$ and stained over-night with $0.025 \%$ Commassie Brilliant Blue G250 (Sigma) in 25\% $\mathrm{MeOH}, 10 \%$ acetic acid, in $\mathrm{H}_{2} \mathrm{O}$. The gels were subsequently destained over-night with $8 \%$ acetic acid and $4 \%$ methanol in $\mathrm{H}_{2} \mathrm{O}$, rocking and at room temperature. Gels were photographed using a Gel Doc ${ }^{\mathrm{TM}}$ XR + System (BioRad).

\subsection{2. $q P C R$}

Total RNA was isolated from cell lines and exosomes using the RNeasy Mini Kit (Qiagen). cDNA was prepared from $48 \mathrm{ng}$ of cell- or exosomes-derived total RNA, respectively, using a High Capacity cDNA Reverse transcription Kit (ABI, UK). Both kits were used according to their manufacturer's instructions. 
mmp-2 (Hs01548724_m1, ABI, UK) and mmp-9 (Hs00957555_m1, ABI, UK) were amplified and threshold cycle $\left(\mathrm{C}_{\mathrm{T}}\right)$ established, using a Mastercycler ${ }^{\circledR}$ ep Realplex (Eppendorf).

\subsection{Statistical analysis}

Statistical analysis was performed using Excel. $p$-Values were generated using Student's $t$-tests. $p<0.05$ was considered to be statistically significant. Results are displayed as $n=3 \pm$ SEM. GraphPad was used for graph generation.

\section{Results}

\subsection{Exosomes were successfully isolated and taken up by recipient/secondary cells}

After performing the isolation procedure, the first step in our analysis was to establish if we had successfully isolated exosomes. To assess this we performed immunoblot analysis to determine the presence of three proteins accepted as markers of exosomes. These include PDC6I/Alix, TSG101 and CD63. We also elected to perform transmission electron microscopy (TEM) analysis to identify if the vesicles isolated were typically with the expected size-range $(30-120 \mathrm{~nm})$ for exosomes. Comparative total protein content was assessed on exosomes expelled from Hs578T and Hs578Ts(i) 8 cells to establish if the quantities released were similar or significantly different. Laser scanning confocal microscopy was performed using samples of fluorescently-labelled exosomes to establish if they could interact with and/ or be taken up by secondary cells to which they are added. Immunoblot analysis for the presence of PDC6I/Alix, TSG101 and CD63 (Fig. 1A) and TEM analysis (Fig. 1B) confirmed successful isolation of Hs578T-exosomes and Hs578Ts(i) $)_{8}$-exosomes. As expected, the proteins assessed were present in the exosome donor cell lines (Fig. 1A). TEM analysis indicated the isolates to be mainly nanosized exosomes $(\sim 100 \mathrm{~nm}$ in diameter) with the characteristic round or 'saucer shape'. Exosome quantities expelled from Hs578T and Hs578Ts(i) $)_{8}$ cells were similar (Fig. 1C). Confocal
A

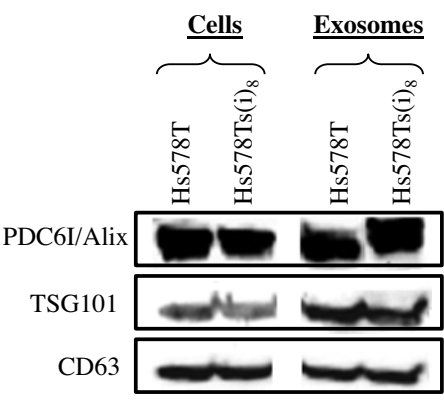

C

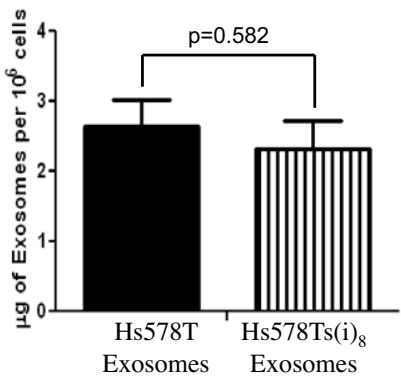

B

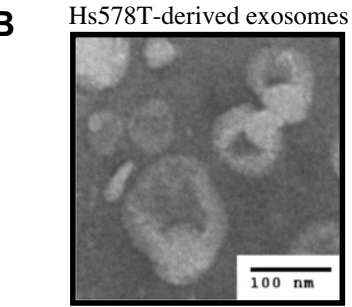

D
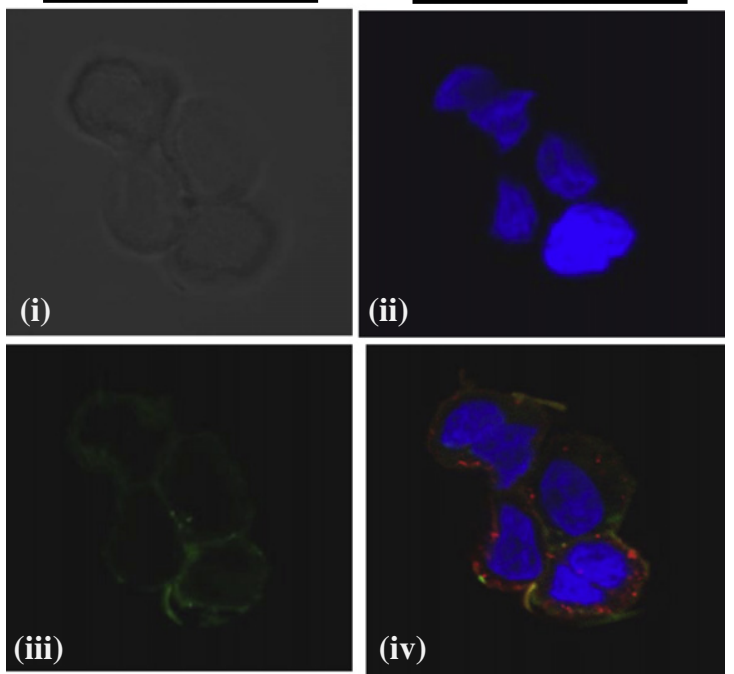

Fig. 1. Successful exosome isolation and their subsequent interaction with secondary cells. Successful isolation of exosomes from medium conditioned by Hs578T and Hs578Ts(i) ${ }_{8}$ cells was confirmed by (A) immunoblot analysis of exosomal markers, PDC6I/Alix, TSG101 and CD63. (B) Transmission electron microscopy showed nano-sized vesicles of 30-100 nm diameter, with a 'saucer like' morphology typical of exosomes $($ scale bar $=100 \mathrm{~nm})$. (C) Exosome protein analysis indicated similar quantities of exosomes to be expelled from Hs578T and Hs578Ts(i) ${ }_{8}$ cells. (D) Confocal microscopy using optical sectioning of cells indicated successful uptake of exosomes by secondary cells with which they were cultured. This image plate illustrates (i) phase contract analysis of SKBR3 (recipient/secondary) cells; (ii) DAPI (blue) staining of SKBR3 cells, showing nuclear region; (iii) cytoplasmic localisation of PKH67GL fluorescently-labelled exosomes (green; Hs578Ts(i) 8 exosomes shown), and (iv) composite image showing exosomes (green) not in the nuclear (blue) region, but in the in cytoplasmic region of SKBR3 cells. (Here SKBR3 cells have been labelled with CM-DiI (red). DiI binds to cellular thiols and is thus used to distinguish the SKBR3 interior and clarify cell structure). (For interpretation of the references to colour in this figure legend, the reader is referred to the web version of this article.) 
microscopy analysis of optically-sectioned cells to which PKH67GL-labelled exosomes had been added (Fig. 1D; SKBR3 shown) confirmed that exosomes are taken into their cytoplasmic region.

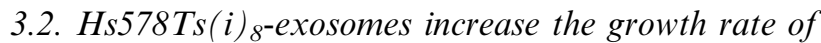 recipient cells}

To investigate if exosomes expelled from these TNBC cell line variants could transfer phenotypic characteristic representative of their cell lines of origin to secondary 'recipient' cells, we assessed the ability of the exosomes to communicate a number of characteristics that differ ${ }^{21}$ between Hs578T and Hs578Ts(i) $)_{8}$ cells. The initial analysis was of growth rate. To ensure that any results obtained were not likely to be recipient cell line-specific but more general, effects on three different secondary cell lines were evaluated. Here Hs578Ts(i $)_{8}$ cells were found to proliferate more rapidly (4.63-fold; $p=0.006$; Fig. 2A) than Hs578T. In keeping with this observation, exosomes expelled from $\mathrm{Hs} 578 \mathrm{Ts}(\mathrm{i})_{8}$ versus $\mathrm{Hs}_{578 \mathrm{~T}}$ cells significantly increased the proliferation of all three recipient cell lines. Specifically, the growth rate of SKBR3 cells was 1.52 -fold $(p=0.016$; Fig. $2 \mathrm{~B}(\mathrm{i}))$ greater in the presence of Hs578Ts(i) $)_{8}$-exosomes compared to Hs578T-exosomes. For MDA-MB-231 cells, the increase was 1.94-fold ( $p=0.018$; Fig. 2B(ii)); for HCC1954, the increase was 1.58 -fold $(p=0.048$; Fig. 2B(iii)).

\subsection{Hs578Ts $(i)_{8 \text {-exosomes increase motility of recipient }}$ cells}

Prior to the studies reported here, using migration inserts $(8 \mu \mathrm{m}$ pore size suitable for use with 24 -well tissue culture plates), applying a fixed number of Hs578T or Hs578Ts(i $)_{8}$ cells into respective inserts and assessing movement through the pores with time - defined as migration - Hughes et al. ${ }^{21}$ had established that the Hs578Ts(i) $)_{8}$ variant cells are 2.5 fold more migratory than Hs578T cells. ${ }^{21}$ In keeping with this trend, here we found Hs578Ts(i) $)_{8}$-exosomes versus Hs578T-exosomes significantly increased the motility of each of the three recipient cell lines evaluated i.e. SKBR3 (2.64-fold, $p=0.033$; Fig. 3A); MDA-MB-231 (3.92fold, $p=0.010$; Fig. 3B); and HCC1954 (1.27-fold, $p=0.001$; Fig. 3C) cells.

\subsection{Hs578Ts $(i)_{8}$-exosomes and MDA-MB-231-exosomes increase invasive potential of recipient cells}

Using a similar approach as for the migration assays, but pre-coating the inserts with ECM, Hs578Ts(i) ${ }_{8}$ had previously been established as approximately three-fold more invasive than Hs578T cells. ${ }^{21}$ Here Hs578Ts(i) $)_{8}$ derived exosomes applied to Hs578T were found to increase the invasion - of the previously less invasive parent cell line - by 1.29 -fold $(p=0.017)$ compared to

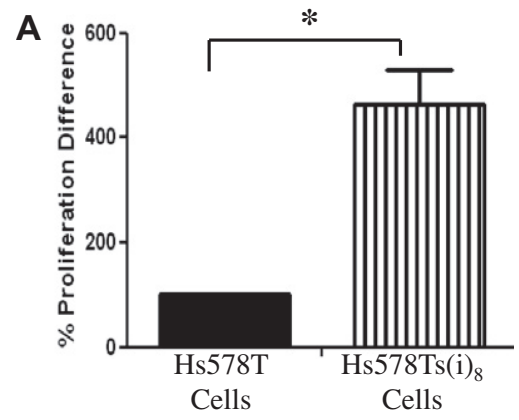

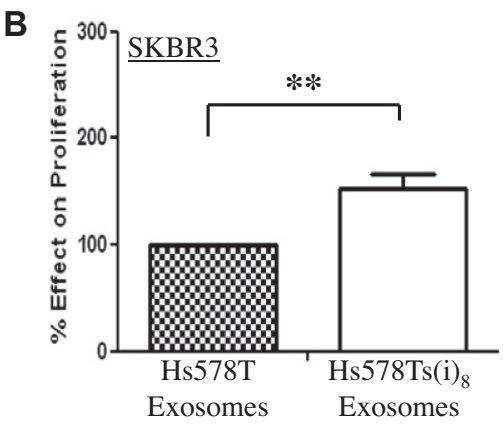

(i)

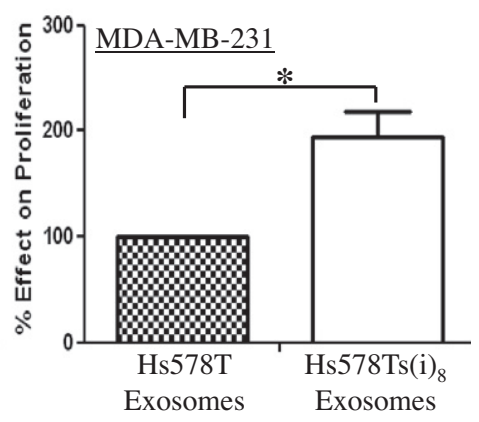

(ii)

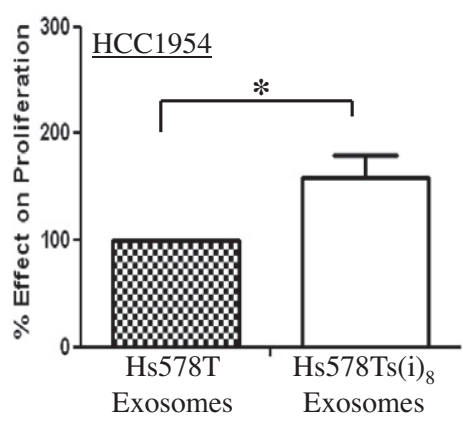

(iii)

Fig. 2. Exosomes affect recipient cells' proliferation in a manner representative of their donor cells' innate characteristics. (A) Hs578Ts(i) ${ }_{8}$ cells were found to grow more rapidly than the parental Hs578T population. (B) Compared to Hs578T-derived exosomes, Hs578Ts(i) - $_{8}$-exosomes significantly increased the proliferation of (i) SKBR3; (ii) MDA-MB-231 and (iii) HCC1954 cells. Results represent triplicate biological repeats, each including three technical repeats and are displayed as mean $\pm \mathrm{SEM}$, where ${ }^{*} p<0.05,{ }^{* *} p<0.01,{ }^{* * *} p<0.001$. 
the invasion of Hs578T cells only exposed to PBS (Fig. 4A). Interestingly, when this analysis was performed using autologous Hs578T-derived exosomes as comparator instead of PBS, the difference between the effects of Hs578Ts(i) $)_{8}$-derived exosomes compared to Hs578T-derived exosomes on Hs578T cells was still significant $(p=0.003)$, but the fold difference was less marked i.e. 1.12-fold (Supplementary Fig. 1). This suggests that a concentrated amount of autologous ('self') exosomes has some influence on their cells of origin, but significantly less effect than that of exosomes expelled from the more invasive Hs578Ts(i $)_{8}$ cells.

In relation to the effects on other recipient cells,

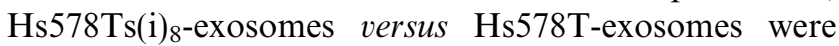
found to significantly increase invasion of SKBR3 (1.42-fold, $\quad p=0.016), \quad$ MDA-MB-231 (1.52-fold, $p=0.0004)$ and HCC1954 (1.19-fold, $p=0.021)$ (Fig. 4B).

In order to determine if these affects are specific to these Hs578T and Hs578Ts(i) $)_{8}$ variants or potentially more broadly relevant, exosomes from another TNBC cell line, MDA-MB-231, were assessed. Following confirmation of their successful isolation (immunoblot: PDC6I/Alix, TSG101, CD63 shown as example results; Fig. 4C(i)), MDA-MB-231-derived exosomes were found to significantly (1.24-fold, $p=0.001)$ increase SKBR3 invasion (Fig. 4C(ii and iii)).

\subsection{MMP-2 and MMP-9 are apparently not transported in substantial quantities via Hs578T- or Hs578Ts $(i)_{8^{-}}$ exosomes}

Matrix metalloproteinases (MMPs) are zinc-dependent endopeptidases capable of degrading extracellular matrix. MMP-2 and MMP-9 secretion is elevated in several types of human cancers and their elevated expression has been associated with poor prognosis. ${ }^{25}$ The typical methods for MMP-2 and MMP-9 activity analysis are via zymography i.e. an electrophoretic technique, based on SDS-PAGE, that includes a substrate co-polymerised with the polyacrylamide gel, to enable detection of enzyme activity. qPCR is also a possibility for assessing corresponding mRNA levels and using Affymetrix microarrays, Hs578Ts(i) $)_{8}$ cells have been shown to contain substantially higher levels of mmp- 2 mRNA compared to that expressed by Hs578T cells. ${ }^{21}$

Here zymography analysis of Hs578T and Hs578Ts(i) 8 cell samples, performed in triplicate, showed significantly higher MMP-2 activity in Hs578Ts(i) $)_{8}$ cells compared to Hs578T cells (2.37 fold;

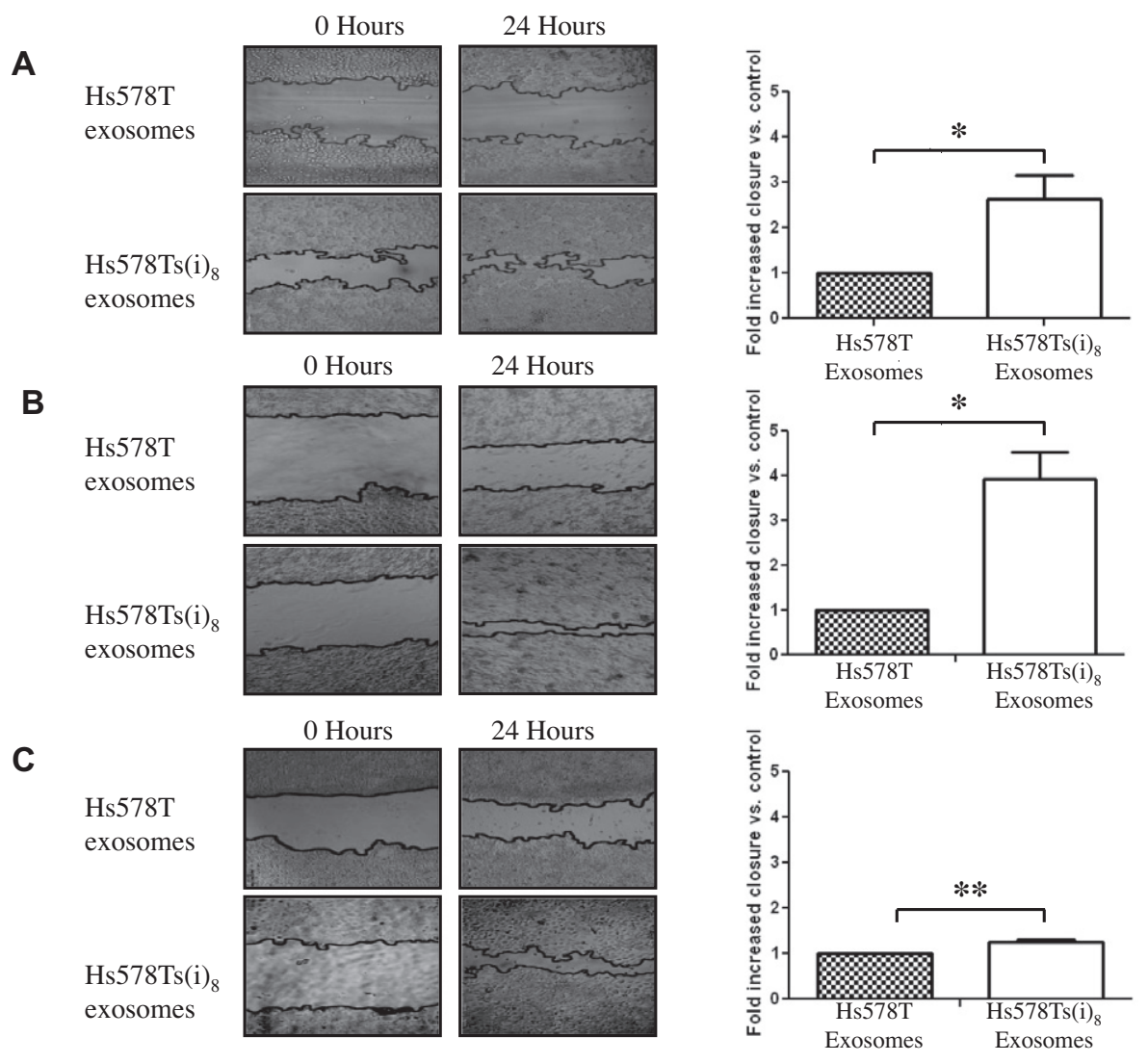

Fig. 3. Exosomes affect recipient cells' motility in a manner representative of their donor cells' innate characteristics. Wound-healing assays showed that exosomes from Hs578Ts(i) $)_{8}$ cells, compared to those from Hs578T cells, significantly increased the motility of A, SKBR3; B, MDA-MB-231 and C, HCC1954 cells. Representative images of wound-healing assays at $0 \mathrm{~h}$ and $24 \mathrm{~h}$ are shown. Graphs represent triplicate biological repeats, each including three technical repeats and are displayed as mean $\pm \mathrm{SEM}$, where ${ }^{*} p<0.05,{ }^{* *} p<0.01,{ }^{* * *} p<0.001$. 
$p=0.0005$ ) (Fig. 5A (i)). MMP-9 enzyme activity was undetected in both cell lines (not shown). The same analysis for MMP-2 using both cell and their respective exosomes samples, confirmed MMP-2 activity in cells; but not in the corresponding exosomes (Fig. 5A(ii)). To determine if the corresponding mRNA was present, qPCR analysis of aliquots of the same samples detected mmp-2 mRNA in Hs578T cells after approximately 34 cycles, while mmp-2 mRNA was detected in Hs578Ts(i) cell samples significantly $(p=0.004)$ earlier (at approximately 30 cycle threshold, $\mathrm{C}_{\mathrm{T}}$ (Fig. 5B). However, assessing the corresponding exosomes, mmp-2 mRNA was undetected in both Hs578T-exosomes and Hs578Ts(i) $)_{8}$-exosomes within 40 cycles. Similarly, mmp-9 mRNA was detected in both cell variants, with significantly $(p=0.004)$ higher levels in Hs578Ts(i) compared to Hs578T cells; albeit levels of mmp-9 mRNA were overall lower than levels of mmp-2 mRNA
(Fig. 5C). As for mmp-2, mmp-9 mRNA was undetected in corresponding exosomes within 40 cycles of qPCR.

\subsection{Exosomes confer anoikis sensitivity/resistance to secondary cells, representing innate characteristics of their cells of origin}

Most breast cancers are of epithelial cells. Epithelial cells typically are attached to a basement membrane, rather than existing in suspension. For such cells to survive in suspension, as required for circulating tumour cells to be transported in the blood stream or lymphatics and progress to forming tumour metastasis, these cells must evade a form of apoptosis termed anoikis. ${ }^{26-28}$ Here, using standard methods, ${ }^{29,30}$ we attempted to mimic this situation in vitro by coating tissue culture plates with p-HEMA and thus inhibiting the ability of the cells to attach to the tissue culture plastic. We subse-
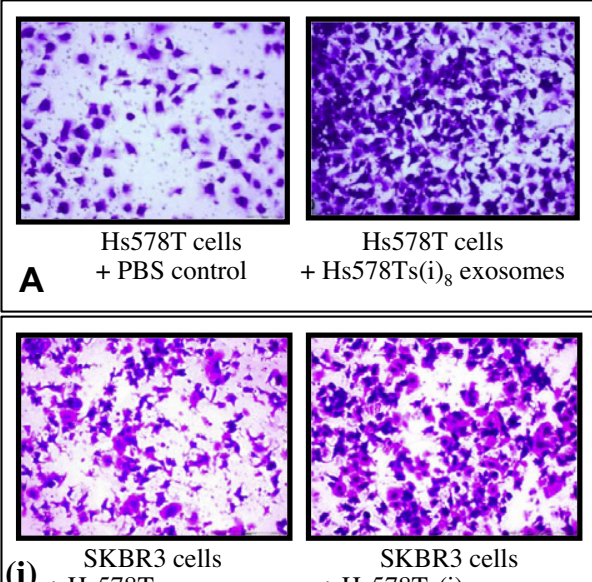

SKBR3 cells
+ Hs578T exosomes

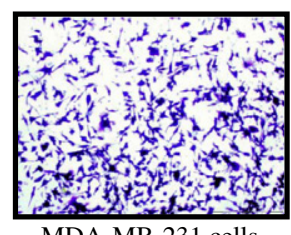

MDA-MB-231 cells (ii) + Hs578T exosomes
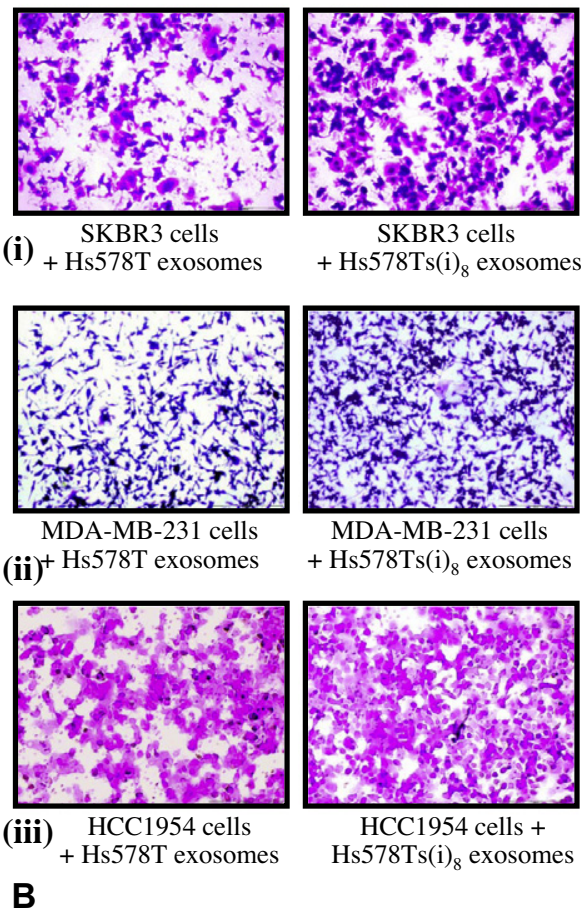

SKBR3 cells

+ Hs578Ts(i) ${ }_{8}$ exosomes
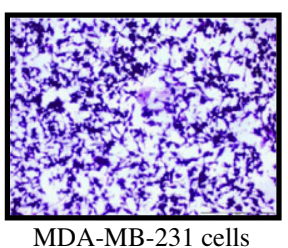

+ Hs578Ts(i) $)_{8}$ exosomes

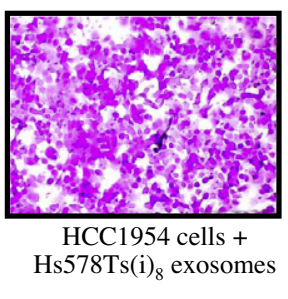

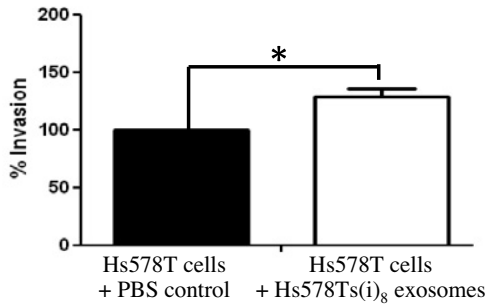

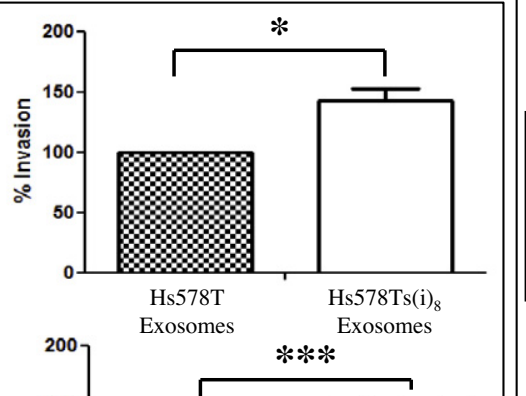

$+\mathrm{Hs} 578 \mathrm{Ts}(1)_{8}$ exosomes

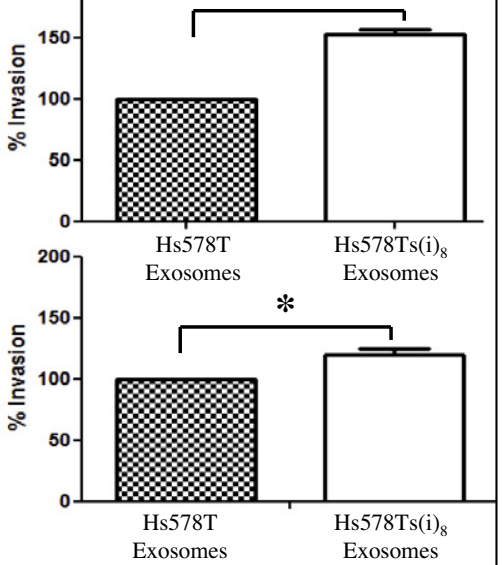

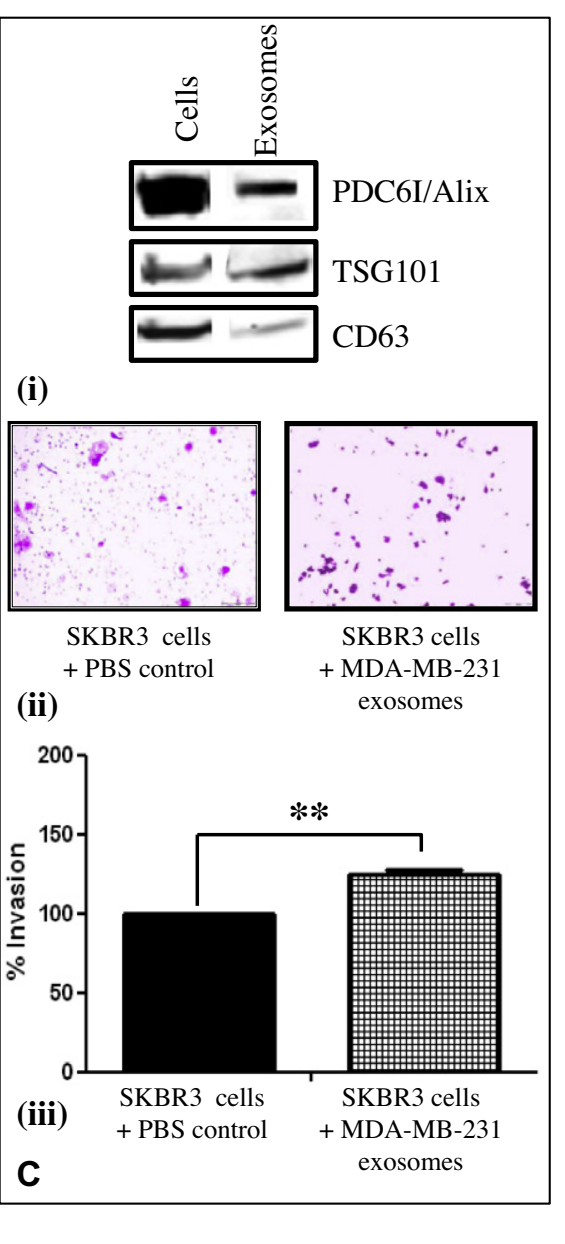

Fig. 4. Exosomes affect recipient cells' invasiveness in a manner representative of their donor cells' innate characteristics. (A) Exosomes from the more invasive Hs578Ts(i) $)_{8}$ clonal population were found to increase the invasion of the parent population, Hs578T. Furthermore, (B) Hs578Ts(i) 8 conferred increased invasion on (i) SKBR3, (ii) MDA-MB-231, and (iii) HCC1954 cells, compared to the affects of Hs578T-derived exosomes. This is not only specific to exosomes from one cell line as shown by (C), data derived from analysis of MDA-MB-231-exosomes when following successful isolation of exosomes ((i) PDC6I/Alix, TSG101 andCD63 immunblot analyses of exosomes shown), these exosomes were found to stimulate recipient cell (SKBR3) invasion when compared to the effects of an equal volume of PBS vehicle control. Representative images of cells that have invaded through extracellular matrix (ECM) after $72 \mathrm{~h}$ are shown. Graphs represent triplicate biological repeats, each including three technical repeats and are displayed as mean \pm SEM, where ${ }^{*} p<0.05,{ }^{* *} p<0.01,{ }^{* * *} p<0.001$. 
quently assessed the ability of the cells to survive i.e. to resist anoikis. Possibly counter-intuitively, Hs578Ts(i)s cells were found to be $14 \%(p=0.009)$ more sensitive to anoikis than Hs578T cells (Fig. 6(A)).

Subsequently determining if this characteristic could be carried to secondary cells via exosomes, we observed that the same trend was communicated i.e. Hs578Ts $(\mathrm{i})_{8}$ exosomes, compared to Hs578T-exosomes, conferred greater anoikis sensitivity to SKBR3 $(13 \%, p=0.006$; Fig. 6B(i)), MDA-MB-231 (9\%, $p=0.017$; Fig. 6B(ii)), and HCC1954 (13\%, $p=0.0001$; Fig 6B(iii)).

\subsection{Hs578Ts $(i)_{8}$-exosomes compared to Hs578T- exosomes stimulate greater vasculogenesis and angiogenesis}

Two distinct mechanisms, vasculogenesis and angiogenesis, implement the formation of vascular networks. Vasculogenesis is the process of new blood vessel formation from endothelial cells. Angiogenesis is the subsequent process by which these vessels take shape from these existing blood vessels by 'sprouting' of endothelial cells thus expanding the vascular tree. ${ }^{31,32}$ For tumour mass survival and continuous growth, tumours must develop a blood vessel network to help carry oxygenated blood, nutrients, etc. to the cancer cells within the tumour mass. So here we were interested in establishing if the exosomes populations under analysis could in anyway influence this phenomenon.

To investigate this, here we used endothelial cells (supplied as V2a Kit; TCS-Cellworks) which we exposed to either Hs578Ts(i) $)_{8}$-derived exosomes or Hs578Tderived exosomes. We assessed the ability of the endothelial cells to form vessels and subsequently expand the network of vessels from these now pre-existing vessels over a period of 2 weeks. These studies indicated that Hs578Ts(i) $)_{8}$-derived exosomes could induce greater (2.67-fold, $p=0.0004)$ angiogenesis after their addition to endothelial cells compared to the affects resulting from exposure to Hs578T-exosomes, as indicated visually (Fig. 7A) and quantitatively (Fig. 7B).

\subsection{Exosomes from TNBC sera, compared to those from healthy control sera, have a stronger stimulatory influence on invasion of secondary cells}

As the studies from our cell lines suggested that exosomes from TNBC cells could communicate (generally
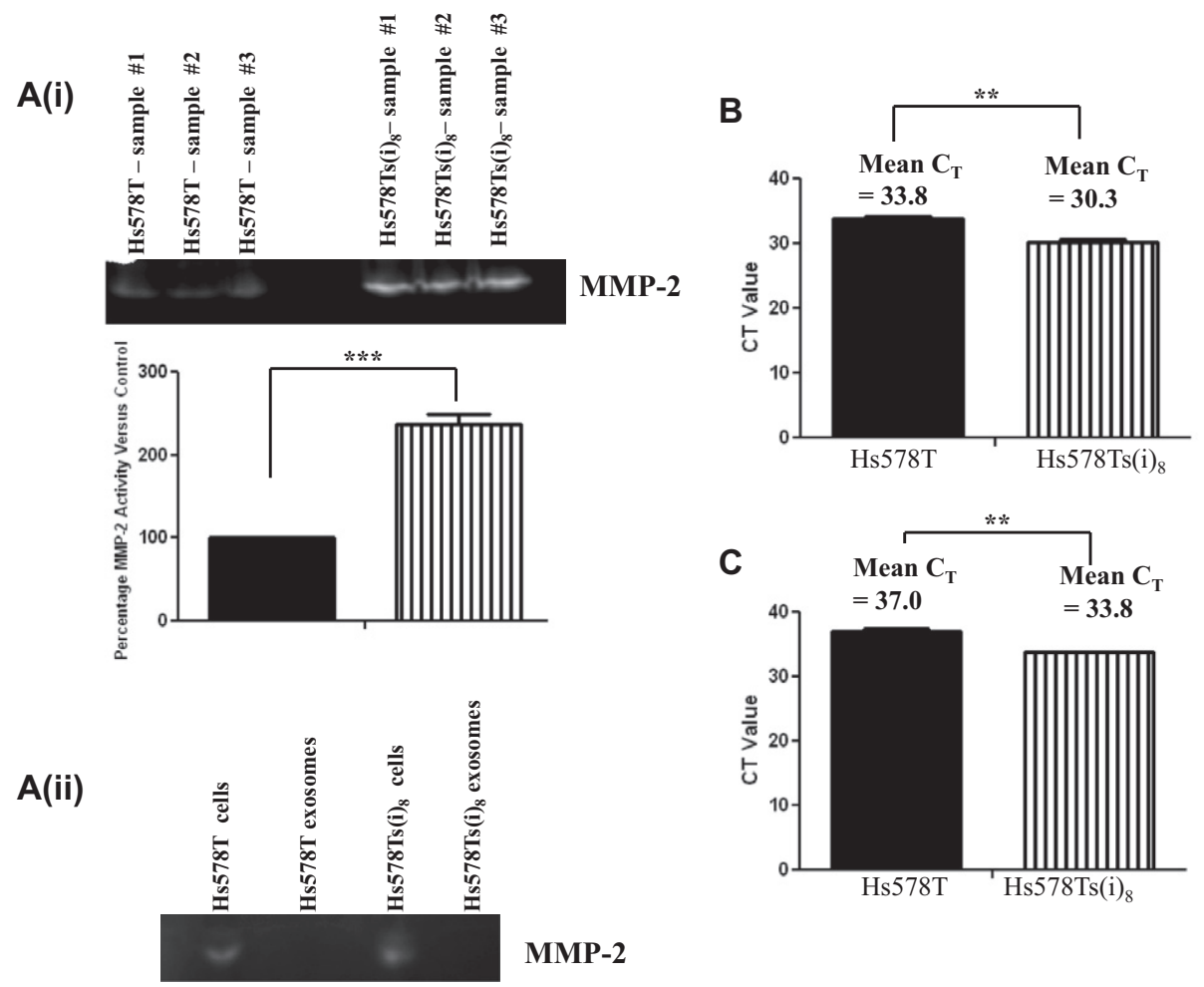

Fig. 5. MMP-2 or MMP-9 in exosomes is apparently not substantial contributor to secondary cell invasion. (A(i)) Zymography analysis of Hs578T and Hs578Ts(i) $)_{8}$ cell samples, showed substantially higher MMP-2 activity in Hs578Ts(i) $)_{8}$ compared to Hs578T (zymogram - top; densitometryderived data-bottom). (A(ii)) The same analysis for MMP-2 using both cell and exosome samples confirmed MMP-2 activity in cells, but not in the corresponding exosomes. qPCR analysis of aliquots of the same samples detected (B), mmp-2 mRNA in Hs578T cells after approximately 34 cycles, while mmp-2 mRNA was detected in Hs578Ts(i) $)_{8}$ cell samples significantly $(p=0.004)$ earlier. mmp-2 mRNA was undetected in corresponding exosomes within 40 cycles. (C) Similarly, mmp-9 mRNA was detected in both cell variants, with significantly $(p=0.004)$ higher levels in Hs578Ts(i $)_{8}$ compared to Hs578T cells. mmp-9 mRNA was undetected in corresponding exosomes within 40 cycles of qPCR. Results are displayed as mean $\pm \mathrm{SEM}$. 
A

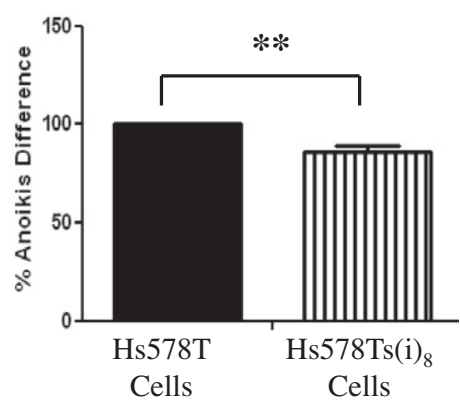

B

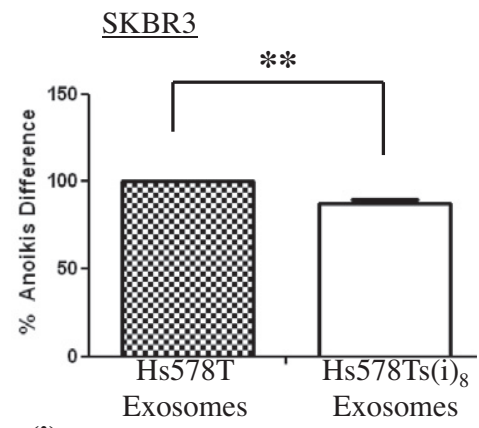

(i)

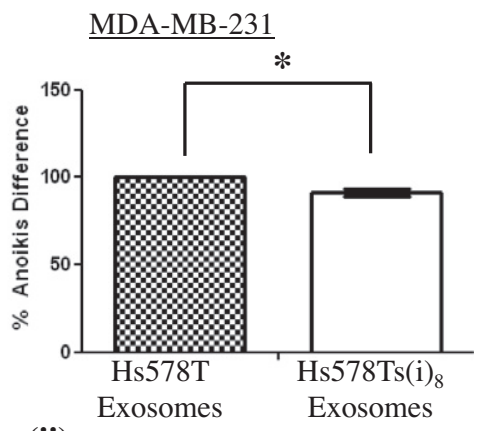

(ii)

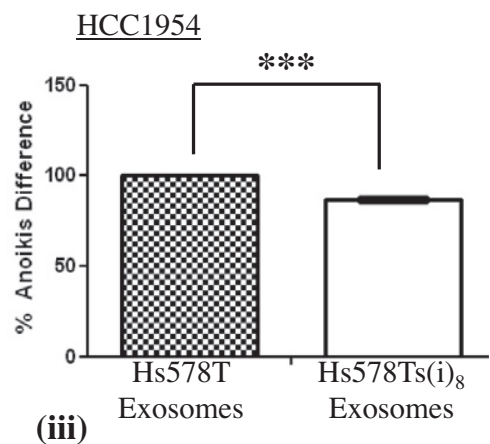

(iii)

Fig. 6. Exosomes affect recipient cell anoikis in a manner representative of their donor cells' innate characteristics. (A) Hs578T cells were found to be more resistant to anoikis than their clonal Hs578Ts(i) ${ }_{8}$ cells. (B) Analyses performed to establish if these populations of exosomes differed in their influences on recipient cells showed that Hs578Ts(i) ${ }_{8}$-derived exosomes conferred greater anoikis sensitivity to recipient cells when compared to the effects of Hs578T-exosomes. This was observed for all cell lines tested (i) SKBR3; (ii) MDA-MB-231 and (iii) HCC1954. Results shown represent triplicate biological repeats and are displayed as mean $\pm \mathrm{SEM}$, where ${ }^{*} p<0.05,{ }^{* *} p<0.01,{ }^{* * *} p<0.001$.

undesirable) phenotypic traits to secondary cells, we felt that it was relevant and important to determine if, in principle, this observation may be clinically relevant. For this proof-of-principle study, we chose to study exosomes derived from the circulation of patients with TNBC compared to age- and gender-matched healthy controls. To achieve this, serum exosomes were successfully isolated from consenting patients with TNBC and from healthy, age-matched volunteers who had no history of cancer. Table 1, summarising information available on the donors, showed no significant differences between any of the known characteristics of the TNBC patients and the healthy control individuals with representative immunoblots for PDC6I/Alix and TSG101 as shown in Fig. 8A, indicating no substantial difference in levels of PDC6I/Alix or TSG101 between TNBC-exosomes and control-exosomes. While the yield of exosomes from TNBC sera tended to be marginally greater than that from control sera, this was not significant (Fig. 8B). However, the TNBC serum-exosomes induced significantly greater (2.23-fold, $p=0.013$ ) invasion of recipient cells, as illustrated in Fig. 8C (SKBR3 included as example model: SKBR3 invasion images (top); quantitative expression of SKBR3 invasion (bottom)). [TNBC serum samples were not pooled to be used, instead each assay was performed 12 independent times, using 12 different TNBC exosomes specimens and analysed against 12 age-matched healthy controls' exosomes specimens].

\subsection{Exosomes from TNBC sera and from healthy control sera contain Claudin-1, -3, -4 and -5 proteins}

Studies, focussing on ovarian cancer, have reported claudin-containing exosomes in the peripheral circulation; with claudin-4 containing exosomes associated with cancer, but not with exosomes from healthy volunteers. ${ }^{33}$ In our pilot study, we detected claudin-1, $-3,-4$ and -5 proteins in all TNBC exosomes. However, we also detected the same trend in all healthy control exosomes (Fig. 9).

\section{Discussion}

A limited number of studies have indicated breast cancer cell-derived exosomes and/or microvesicles to have functional relevance. Lau and Wong ${ }^{19}$ assessed the effects of MDA-MB-231 'exosome-like microvesicles' on human submandibular gland (HSG) cells, using lysed exosome-like microvesicles as controls. Intact exosome-like particles, compared to controls, increased the total amount of RNA in HSG cells and resulted in increased levels of 88 proteins in subsequent HSGderived exosome-like particles. Of particular relevance to HER2-positive breast cancers, exosomes from HER2-overexpressing breast cell lines, BT474 and SKBR3 (compared to those from melanoma cells, as control), sequestered the therapeutic antibody trastuzumab and thus substantially reduced its binding to 
A

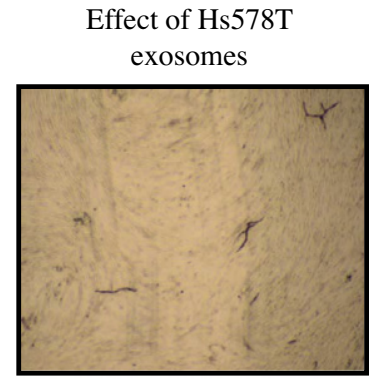

Effect of Hs578Ts(i) exosomes

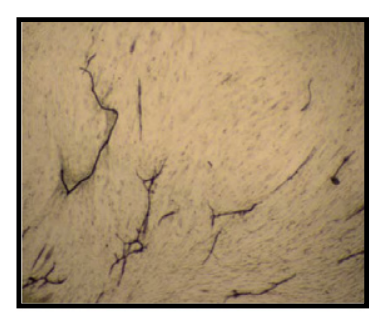

B

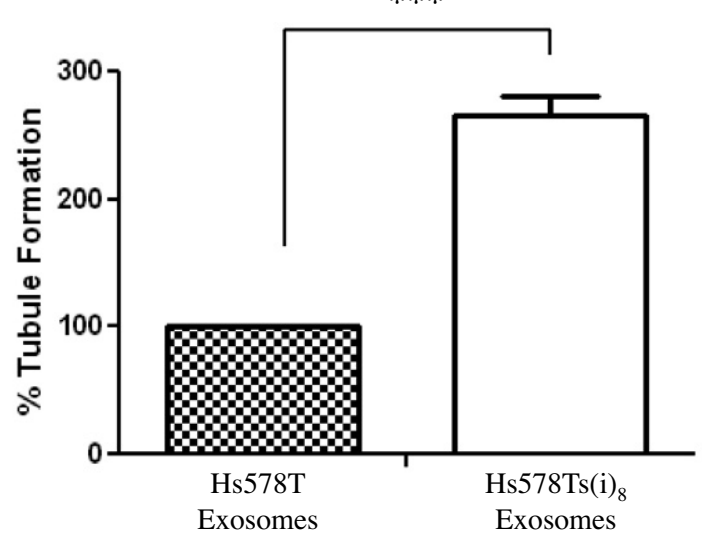

Fig. 7. Hs578Ts(i) $)_{8}$-exosomes compared to Hs578T-exosomes stimulate greater vasculogenesis/angiogenesis. In vitro vasculogenesis/angiogenesis assays, performed in triplicate, indicated that Hs578Ts(i) $8_{8^{-}}$ exosomes stimulated significantly more endothelial tubule formation compared to the response of these cells to Hs578T-exosomes. This is shown as (A) images of the tubule formation 14 days after exosomes exposure, and (B) graphically, representing the quantitative effects. Results are displayed as mean $\pm \mathrm{SEM}$, where ${ }^{* * *} p<0.001$.

tumour cells, with an overall effect of reducing in vitro antibody-dependent cellular cytotoxicity. ${ }^{18}$ In their associated study of serum from patients $(n=12)$ with invasive breast cancer, approximately one-third of the serum HER2 was found to be exosome-associated. Advancing on this, exosomes from HER2-positive (SKBR3, BT474), but not HER2-negative (MDA-MB231), cells decreased trastuzumab's anti-proliferative effects on SKBR3 cells, while the effect of lapatinib (targeting epidermal growth factor receptor (EGFR) and HER2 was unaffected). ${ }^{18}$

In cancer, it is typically the metastases that result in death. Metastasis is a multi-step event involving invasion at the primary site, intravasation of tumour cells through ECM to either blood or lymphatic vessels to circulate. These cells must be able to subsequently extravasate at the distant organ and establish secondary tumours or metastases. Recent studies indicate that exosomes may be involved in preparing the pre-metastatic niche and subsequently attracting cancer cells to that site, further highlighting the importance of elucidating their potential role in cancer and cancer metastasis. ${ }^{34,35}$ As TNBC is a particular problematic 'unmet clinical need', frequently presenting with aggressive behaviour and relapsing with distant metastases rather than with local sites, we believe that it is timely to commence studies investigating the possible relevance of exosomes in this disease.

Hs578Ts(i) ${ }_{8}$ is an isogenic subclone of the TNBC cell line, Hs578T. Prior to this study, Hs578Ts(i) 8 was identified and characterised by co-authors Hughes, Mc Donnell and others. ${ }^{21}$ Hs578Ts(i) ${ }_{8}$ is significantly more proliferative $(p<0.019)$, motile $(p<0.001)$ and invasive $(p<0.0002)$ than Hs578T cells. Here our initial analyses were focussed on determining if exosomal phenotypic affects on secondary cells would reflect the cellular origin of the Hs578T- and Hs578Ts(i) $)_{8}$-derived exosomes, respectively. In brief, having established that each of these three phenotypic differences was conferred on all three recipient cell lines tested, we subsequently progress to assess exosomal influence on recipient cell anoikis and also on tubule formation by endothelial cells (mimicking, in as much as possible, events necessary for neovascularisation and subsequent angiogenesis).

Of note to highlight, a concern with studies aimed at elucidating the functional relevance of exosomes is identifying a suitable control. Depending on the scientific question being asked, exosomes from cells lacking a specific receptor (examples above) may be relevant. For other studies, saline/PBS or culture medium is used, but raise the concern of being unable to control for potential physical or mechanical effects, in addition to the biological effects of these nano-sized entities. Recent studies have involved generating nano-sized liposomes specifically for this purpose. ${ }^{34}$ Here, for the main parts of our analysis, we had the opportunity to compare exosomes isolated - in parallel and under precisely the same conditions - from matching isogenic populations, to assess if phenotypic characteristics established as differing significantly between the cell populations would be passed on to secondary 'recipient' cells. As indicated, TEM and immunoblotting confirmed successful isolation of vesicles of the expected size, shape and markers indicative of exosomes. As typical for reported studies of exosomes, we cannot definitely claim a complete absence of microvesicles that are not exosomes. If so, however, this is likely to be consistent in both the Hs578T and Hs578Ts(i) $)_{8}$ isolates and, for this study, we were most interested in knowing if epigenetic information can be relayed and transfer phenotypic changes to recipient cells. To ensure that any observations made by analysing the effects of these exosomes were not specific to one recipient cell line, all subsequent analysis of these exosomes included three secondary recipient cell lines, SKBR3, MDA-MB-231 and HCC1954.

Confocal microscopy analysis of labelled exosome populations indicated their successful interaction/ uptake by secondary cells. Without exception, differential characteristics (increased proliferation, motility and invasion) established between the Hs578Ts(i) ${ }_{8}$ and 


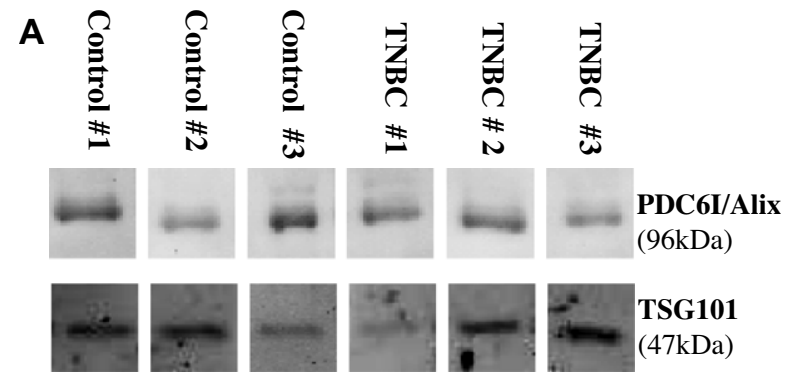

C
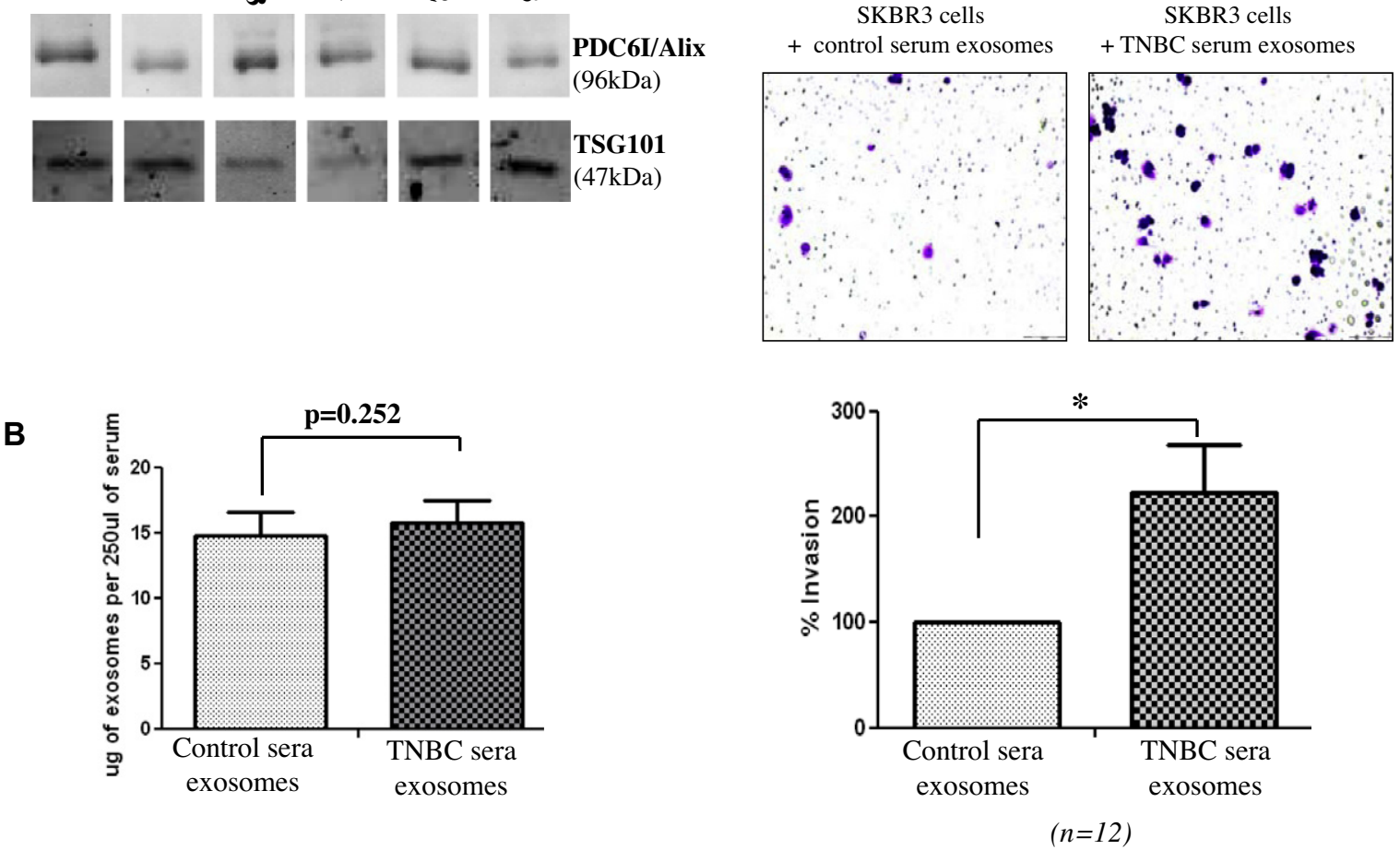

Fig. 8. Effects of exosomes from triple-negative breast cancer (TNBC) sera compared to those from healthy control. This proof-of-principle translational study showed successful isolation of exosomes from serum. (A) Representative immunoblot for exosomal markers PDC6I/Alix and TSG101. (B) Protein quantitation of aliquots of exosomes isolated from each serum specimens showed no significant differences in quantities of exosomes in TNBC and healthy control serum. C, exosomes from TNBC sera ( $n=12$ individually analysed) significantly increased cellular invasion (SKBR3 shown), when compared to the affects of exposing the same cells to exosomes from healthy control sera ( $n=12$, age matched). (C (top)) Representative images of cells that have invaded through ECM after $72 \mathrm{~h}$ are shown; (C (bottom)) graph represents quantitative results following crystal violet elution and absorbance assessment shown as mean $\pm \mathrm{SEM}$, where ${ }^{*} p<0.05$.

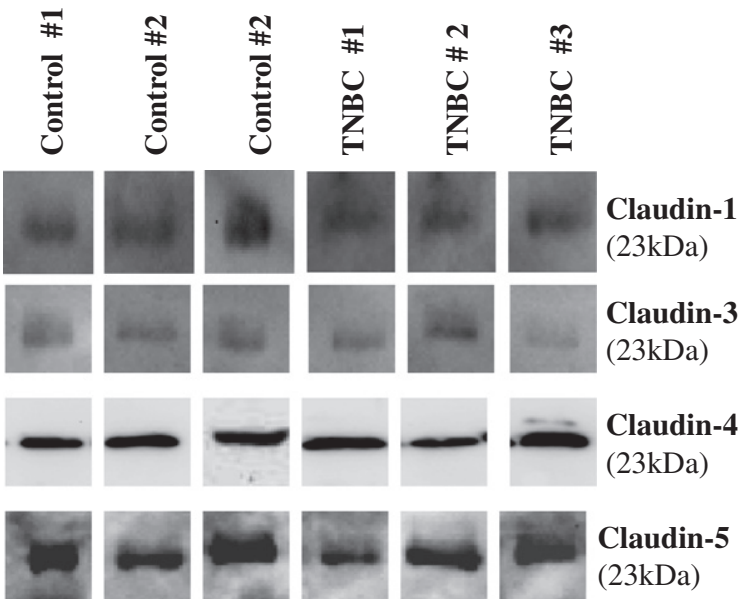

Fig. 9. Claudin proteins in exosomes from TNBC and control serum specimens. Immunoblot analysis for exosomal claudin-1, -3, -4 and -5 (showing representative exosome analysis for TNBC and healthy controls $(n=3$ each), respectively, showed all four claudins to be present in all specimens, with no substantial difference in levels between TNBC and control exosomes.
Hs578T populations were all portrayed by each of the three recipient cells. While we cannot claim precisely what may be contributing to this difference on recipient cell phenotype, it is noteworthy that $\operatorname{Hs} 578 \mathrm{Ts}(\mathrm{i})_{8}$ cells have $30 \%$ more $\mathrm{CD} 44^{+} / \mathrm{CD} 24^{- \text {low }}$ cells than the parent Hs578T cells. ${ }^{21} \mathrm{CD} 44^{+} / \mathrm{CD} 24^{-/ \text {low }}$ is the accepted phenotype marker set characterising breast cancer stem cells (BCSCs) attributed with features that are demonstrated by the disease itself, such as growth of tumour, recurrence, metastases. ${ }^{36,37}$ So we hypothesised that exosomes derived from the $\mathrm{CD} 44^{+} / \mathrm{CD} 24^{-/ \text {low }}$ population of $\mathrm{Hs} 578 \mathrm{Ts}(\mathrm{i})_{8}$ may be substantially contributing to this difference. Future studies including exosomes derived from the Hs578Ts $(\mathrm{i})_{8} \mathrm{CD} 44^{+} / \mathrm{CD} 24^{-/ \text {low }}$ cells compared to exosomes from the remaining approximately $70 \%$ Hs578Ts(i) 8 population will help to support (or otherwise) this hypothesis.

MMPs are involved in degrading extracellular matrix, necessary for invasion. So here we elected to study these enzymes in Hs578T and Hs578Ts(i) 8 variants and their corresponding exosomes. MMP-2, but not MMP-9, enzyme activity was detected in Hs578T 
and Hs578Ts(i $)_{8}$ cell samples, with significantly greater MMP-2 activity in Hs578Ts(i) 8 . Neither MMP-2 nor MMP-9 activity was detected in their corresponding exosomes. Furthermore, subsequent qPCR analyses indicated mmp- 2 and mmp- 9 mRNA present within Hs578T and Hs578Ts(i) $)_{8}$ cells, but neither transcript was detected in the corresponding exosomes - using the same quantity of starting total RNA and up to 40 cycles of real-time PCR.

Hs578Ts(i) $)_{8}$ were found to be more sensitive to anoikis than the parental Hs578T cells. This is somewhat unexpected, due to their generally otherwise more 'aggressive' nature. Interestingly, a similar observation was seen when assessing anoikis resistance in $\mathrm{LNCaP}$ isogenic variant cells. ${ }^{38}$ Briefly, two isogenic variants were isolated from LNCap cells i.e. LNCaP-LN-4, a highly-metastatic variant and LNCaP-PRO-5, a nonmetastatic variant. When analysing the anoikis resistance of the variants against each other, they observed that the highly-metastatic LNCaP-LN-4 were at least as sensitive to anoikis as the non-metastatic LNCaPPRO-5 cells. While we are not in a position to speculate as to why this might be, this apparent disconnect between invasion and anoikis may be of interest to further investigate in subsequent studies.

While it was not possible, with exosomes, to model the increased ability of $\mathrm{Hs} 578 \mathrm{Ts}(\mathrm{i})_{8}$ cells to produce tumours in mice, it is noteworthy that Hs578Ts(i) $)_{8}$-exosomes stimulated significantly more endothelial tubule formation, a characteristic that would support tumour growth and facilitate interactions of tumour cells with the microenvironment.

To assess if the phenotypic observations made with Hs578T- and Hs578Ts(i) $)_{8}$-exosomes are not simply a cell line-related phenomenon, but may have clinical relevance in TNBC, we performed a proof-of-principle study of exosomes isolated from relevant serum specimens. This pilot study of exosomes from TNBC patients compared to healthy controls found no substantial differences in exosome quantities between these populations of exosomes. This difference from observations made with ovarian cancer sera versus control sera, ${ }^{39}$ may be cancer-type specific (breast cancer is frequently diagnosed early when the tumour is small; ovarian cancer is frequently diagnosed when the tumour is at a more advanced stage), methods of isolation and methods of quantification. To investigate this further, larger studies co-analysing and comparing larger cohorts of many cancers and control specimens are warranted. In this study, using the invasion assay approach as relevant starting point because of the fundamental importance of this step in metastasis, showed that TNBC-derived exosomes stimulate significantly greater invasion by secondary cells.

As claudin- 4 has been associated with exosomes from patients with ovarian cancer (32/63 plasma-derived exo- somes samples analysed), but not with exosomes from healthy volunteers (only detected in 1 of 50 exosome specimens analysed $)^{33}$, here we chose to assess if any or all of claudin-1, $-3,-4$ and -5 proteins would show differential presence in exosomes from TNBC patients and controls. Our results indicated all 4 claudins analysed to be present in all (TNBC and control) exosomes. Possible reasons for the discrepancy between the healthy control exosomes in this study and those in the previously reported ovarian cancer study may be due to difference in antibodies used. Li et al. ${ }^{33}$ assessed claudin-4 in conditioned media-derived exosomes with an antibody from one supplier (i.e. this antibody, sourced from Invitrogen, was the antibody we consistently used for all our studies) but, for reasons that are unclear to us, they elected to use any antibody from a different supplier (Abcam) for their peripheral circulation exosome analysis. An alternative explanation may be the differences in exosome quantities used in the immunoblots. Here we performed our assessment of exosomes by separating a fixed amount (based on protein analysis; $10 \mu \mathrm{g}$ ) from each exosomal specimen. While Li et al. ${ }^{33}$ re-suspended their isolated exosomes in a fixed volume and assessed on volume fixed basis. Significantly higher levels of exosomes have been reported in the circulation of ovarian cancer patients compared to controls, ${ }^{39}$ but we have not detected substantial higher levels of exosomes in serum from TNBC patients compared to controls, the difference in loading by fixed quantity or loading by fixed volume may also help explain the differing observation made.

In conclusion, in what we understand to be the first study of exosomes in the context of TNBC, here we provide support for a potential role of exosomes in cell-cell transfer of epigenetic information, resulting in phenotypic traits of donor cells being conferred to recipient cells. These results support our hypothesis that, in TNBC, cellular communication via exosomes may - at least in part confer aggressive behaviour to secondary cells. Future studies focused on identifying the specific molecules involved (by profiling the contents of these exosomes) and including analysis of larger cohorts of serum specimens are warranted to expand our understanding of the functional relevance of exosomes in TNBC.

\section{Role of funding source}

Student scholarships, test materials, equipment, etc. are supported by these funds. The sponsors had no involvement in the study design through to manuscript preparation.

\section{Conflict of interest statement}

None of the authors have any conflict of interest to declare. 


\section{Acknowledgements}

The authors wish to thank Mr. Neal Leddy Chief Technical Officer Specialist for his microscopy expertise. We also gratefully acknowledge funding support in the form of the Marie Keating Foundation PhD Scholarship at Trinity College Dublin (LOD); Trinity Foundation, Trinity College Dublin (LOD); Science Foundation Ireland's funding of Strategic Research Cluster, Molecular Therapeutics for Cancer Ireland 08/SRC/B1410 (JC, LOD); the Irish Research Council for Science, Engineering \& Technology (RW, LOD) and the Higher Education Authorities Programme for Research in Third-Level Institutions Cycle 5 support for Trinity Biomedical Sciences Institute (MWR, LOD). The sponsors had no involvement in the study design through to manuscript preparation.

\section{Appendix A. Supplementary data}

Supplementary data associated with this article can be found, in the online version, at http://dx.doi.org/ 10.1016/j.ejca.2013.01.017.

\section{References}

1. Gyorgy B, Szabo TG, Pasztoi M, et al. Membrane vesicles, current state-of-the-art: emerging role of extracellular vesicles. Cell Mol Life Sci 2011;68:2667-88.

2. Valadi H, Ekström K, Bossios A, Sjöstrand M, Lee JJ, Lötvall JO. Exosome-mediated transfer of mRNAs and microRNAs is a novel mechanism of genetic exchange between cells. Nat Cell Biol 2007;9:654-9.

3. Skog J, Würdinger T, van Rijn S, et al. Glioblastoma microvesicles transport RNA and proteins that promote tumour growth and provide diagnostic biomarkers. Nat Cell Biol 2008;10:1470-6.

4. Thery C. Exosomes: secreted vesicles and intercellular communications. F1000 Biol Rep 2011;3:15.

5. Pap E, Pallinger E, Falus A. The role of membrane vesicles in tumorigenesis. Crit Rev Oncol Hematol 2011;79:213-23.

6. Ge R, Tan E, Sharghi-Namini S, Asada HH. Exosomes in cancer microenvironment and beyond: have we overlooked these extracellular messengers? Cancer Microenviron 2012;5:323-32.

7. Kharaziha P, Ceder S, Li Q, Panaretakis T. Tumor cell-derived exosomes: a message in a bottle. Biochim Biophys Acta 2012;1826:103-11.

8. Ludwig AK, Giebel B. Exosomes: small vesicles participating in intercellular communication. Int J Biochem Cell Biol 2012;44:11-5.

9. Vlassov AV, Magdaleno S, Setterquist R, Conrad R. Exosomes: current knowledge of their composition, biological functions, and diagnostic and therapeutic potentials. Biochim Biophys Acta 2012;1820:940-8.

10. Perou CM, Sorlie T, Eisen MB, et al. Molecular portraits of human breast tumours. Nature 2000;406:747-52.

11. Carey LA. Through a glass darkly: advances in understanding breast cancer biology, 2000-2010. Clin Breast Cancer 2010; 10:188-95.

12. Anders CK, Carey LA. Biology, metastatic patterns, and treatment of patients with triple-negative breast cancer. Clin Breast Cancer 2009;9:S73-81

13. Lin NU, Claus E, Sohl J, Razzak AR, Arnaout A, Winer EP. Sites of distant recurrence and clinical outcomes in patients with metastatic triple-negative breast cancer: high incidence of central nervous system metastases. Cancer 2008;113:2638-45.

14. Dent R, Hanna WM, Trudeau M, Rawlinson E, Sun P, Narod SA. Pattern of metastatic spread in triple-negative breast cancer. Breast Cancer Res Treat 2009;115:423-8.

15. Arnedos M, Bihan C, Delaloge S, Andre F. Triple-negative breast cancer: are we making headway at least? Ther Adv Med Oncol 2012;4:195-210.

16. Perou CM. Molecular stratification of triple-negative breast cancers. Oncologist 2011;16:61-70.

17. Battke C, Ruiss R, Welsch U, et al. Tumour exosomes inhibit binding of tumour-reactive antibodies to tumour cells and reduce ADCC. Cancer Immunol Immunother 2011;60:639-48.

18. Ciravolo V, Huber V, Ghedini GC, et al. Potential role of HER2overexpressing exosomes in countering trastuzumab-based therapy. J Cell Physiol 2012;227:658-67.

19. Lau CS, Wong DT. Breast cancer exosome-like microvesicles and salivary gland cells interplay alters salivary gland cellderived exosome-like microvesicles in vitro. PLoS One 2012;7:e33037.

20. Prat A, Parker JS, Karginova O, et al. Phenotypic and molecular characterization of the claudin-low intrinsic subtype of breast cancer. Breast Cancer Res 2010;12:R68.

21. Hughes L, Malone C, Chumsri S, Burger AM, McDonnell S. Characterisation of breast cancer cell lines and establishment of a novel isogenic subclone to study migration, invasion and tumourigenicity. Clin Exp Metastasis 2008;25: 549-57.

22. Rani S, O'Brien K, Kelleher FC, et al. Isolation of exosomes for subsequent mRNA, MicroRNA, and protein profiling. Methods Mol Biol 2011;784:181-95.

23. Germano S, Kennedy S, Rani S, et al. MAGE-D4B is a novel marker of poor prognosis and potential therapeutic target involved in breast cancer tumorigenesis. Int $J$ Cancer 2012;130:1991-2002.

24. Wang J, O'Sullivan S, Harmon S, et al. Design of barbituratenitrate hybrids that Inhibit MMP-9 activity and secretion. $J$ Med Chem 2012;55:2154-62.

25. Roomi MW, Monterrey JC, Kalinovsky T, Rath M, Niedzwiecki A. Patterns of MMP-2 and MMP-9 expression in human cancer cell lines. Oncol Rep 2009;21:1323-33.

26. Grossmann J. Molecular mechanisms of "detachment-induced apoptosis - Anoikis”. Apoptosis 2002;7:247-60.

27. Zhan M, Zhao H, Han ZC. Signalling mechanisms of anoikis. Histol Histopathol 2004;19:973-83.

28. Reddig PJ, Juliano RL. Clinging to life: cell to matrix adhesion and cell survival. Cancer Metastasis Rev 2005;24: 425-39.

29. Valentinis B, Morrione A, Peruzzi F, Prisco M, Reiss K, Baserga R. Anti-apoptotic signaling of the IGF-I receptor in fibroblasts following loss of matrix adhesion. Oncogene 1999;18:1827-36.

30. Noda T, Iwai S, Hamada M, Fujita Y, Yura Y. Induction of apoptosis of detached oral squamous cell carcinoma cells by safingol. Possible role of Bim, focal adhesion kinase and endonuclease G. Apoptosis 2009;14:287-97.

31. Kioi M, Vogel H, Schultz G, Hoffman RM, Harsh GR, Brown JM. Inhibition of vasculogenesis, but not angiogenesis, prevents the recurrence of glioblastoma after irradiation in mice. $J$ Clin Invest 2010;120:694-705.

32. Simionescu DT, Simionescu A. Vasculogenesis and angiogenesis from embryonic development to regenerative medicine. New York: InTech-Open Access Publisher; 2011.

33. Li JH, Sherman-Baust CA, Tsai-Turton M, Bristow RE, Roden $\mathrm{RB}$, Morin PJ. Claudin-containing exosomes in the peripheral circulation of women with ovarian cancer. BMC Cancer 2009;9:244. 
34. Hood JL, San RS, Wickline SA. Exosomes released by melanoma cells prepare sentinel lymph nodes for tumor metastasis. Cancer Res 2011;71:3792-801.

35. Peinado $\mathrm{H}$, Aleckovic $\mathrm{M}$, Lavotshkin $\mathrm{S}$, et al. Melanoma exosomes educate bone marrow progenitor cells toward a prometastatic phenotype through MET. Nat Med 2012;18:883-91.

36. Gangopadhyay S, Nandy A, Hor P, Mukhopadhyay A. Breast cancer stem cells: a novel therapeutic target. Clin Breast Cancer 2012;:S1526-8209:00221-2.
37. Wicha MS. Migratory gene expression signature predicts poor patient outcome: are cancer stem cells to blame? Breast Cancer Res 2012;14:114.

38. Bondar VM, McConkey DJ. Anoikis is regulated by BCL-2independent pathways in human prostate carcinoma cells. Prostate 2002;51:42-9.

39. Taylor DD, Gercel-Taylor C, Parker LP. Patient-derived tumorreactive antibodies as diagnostic markers for ovarian cancer. Gynecol Oncol 2009;115:112-20. 